\title{
Variants of normality and their duals: a pointfree unification of insertion and extension theorems for real-valued functions
}

\author{
Javier Gutiérrez García ${ }^{1 *}$ and Jorge Picado ${ }^{2 \dagger}$ \\ 1 Dept. of Mathematics, Univ. of the Basque Country UPV/EHU, Bilbao, Spain \\ javier.gutierrezgarcia@ehu.es \\ ${ }^{2}$ CMUC, Dept. of Mathematics, Univ. of Coimbra, Coimbra, Portugal \\ picado@mat.uc.pt
}

Several pairs of results in classical topology like those in Table 1 characterizing the concepts of normality and extremal disconnectedness show a remarkable duality between the two concepts. Nevertheless the known proofs of the results in each pair are quite different in nature, requiring even in some cases different approaches and tools.

\begin{tabular}{|c|c|c|}
\hline Space $X$ & NORMAL & Extremally Disconnected \\
\hline $\begin{array}{l}\text { Urysohn's } \\
\text { separation type } \\
\text { lemma }\end{array}$ & $\begin{array}{l}\text { Every two disjoint CLOSED } \\
\text { subsets of } X \text { are completely } \\
\text { separated (Urysohn 1925). }\end{array}$ & $\begin{array}{l}\text { Every two disjoint OPEN subsets } \\
\text { of } X \text { are completely separated } \\
\text { (Gillman \& Jerison 1960). }\end{array}$ \\
\hline $\begin{array}{l}\text { Tietze's extension } \\
\text { type lemma }\end{array}$ & $\begin{array}{l}\text { Each CLOSED subset of } X \text { is } \\
C^{*} \text {-embedded (Tietze } 1915 \text { ). }\end{array}$ & $\begin{array}{l}\text { Each OPEN subset of } X \text { is } \\
C^{*} \text {-embedded (Gillman \& Jerison } \\
\text { 1960). }\end{array}$ \\
\hline $\begin{array}{l}\text { Katětov-Tong } \\
\text { insertion type } \\
\text { theorem }\end{array}$ & $\begin{array}{l}\text { For every UPPER semicontinuous } \\
\text { real function } f \text { and LOWER sem- } \\
\text { icontinuous real function } g \text { satis- } \\
\text { fying } f \leq g \text {, there exists a con- } \\
\text { tinuous real function } h \text { such that } \\
f \leq h \leq g \text { (Katětov 1951, Tong } \\
1952 \text { ). }\end{array}$ & $\begin{array}{l}\text { For every LOWER semicontinuous } \\
\text { real function } f \text { and UPPER sem- } \\
\text { icontinuous real function } g \text { satis- } \\
\text { fying } f \leq g \text {, there exists a con- } \\
\text { tinuous real function } h \text { such that } \\
f \leq h \leq g \text { (Stone } 1949 \text {, Lane } \\
1975) \text {. }\end{array}$ \\
\hline
\end{tabular}

Table 1: Characterizations of normal and extremally disconnected spaces

Our recent work in the more general pointfree setting [2, 3, 4] reveals a similar picture, summarized in Table 2 ,

This shapes the idea that the two notions are somehow dual to each other and therefore may be studied in parallel; hopefully, one may even find "dual" proofs for each pair of results.

\footnotetext{
*Research supported by the Ministry of Economy and Competitiveness of Spain (under grant MTM201237894-C02-02) and the UPV/EHU (under grants UFI11/52 and GIU12/39).

${ }^{\dagger}$ Research supported the Centre for Mathematics of the University of Coimbra (funded by the European Regional Development Fund through the program COMPETE and by the Portuguese Government through the Fundação para a Ciência e a Tecnologia, under the project PEst-C/MAT/UI0324/2011).
} 


\begin{tabular}{lll}
\hline Locale $\boldsymbol{L}$ & NORMAL & EXTREMALLY DISCONNECTED \\
\hline $\begin{array}{l}\text { Urysohn's } \\
\text { separation type } \\
\text { lemma }\end{array}$ & $\begin{array}{l}\text { Every two disjoint CLOSED } \\
\text { sublocales of } L \text { are completely } \\
\text { separated. }\end{array}$ & $\begin{array}{l}\text { Every two disjoint OPEN } \\
\text { sublocales of } L \text { are completely } \\
\text { separated. }\end{array}$ \\
\hline $\begin{array}{l}\text { Tietze's extension } \\
\text { type lemma }\end{array}$ & $\begin{array}{l}\text { Each CLOSED sublocale of } L \text { is } \\
C^{*} \text {-embedded. }\end{array}$ & $\begin{array}{l}\text { Each OPEN sublocale of } L \text { is } \\
C^{*} \text {-embedded. }\end{array}$ \\
\hline $\begin{array}{l}\text { Katětov-Tong } \\
\text { insertion type } \\
\text { theorem }\end{array}$ & $\begin{array}{l}\text { For every UPPER semicontinuous } \\
\text { real function } f \text { and LOwER sem- }\end{array}$ & $\begin{array}{l}\text { For every LOWER semicontinuous } \\
\text { real function } f \text { and UPPER sem- }\end{array}$ \\
& $\begin{array}{l}\text { icontinuous real function } g \text { satis- } \\
\text { icontinuous real function } g \text { satis- } \\
\text { fying } f \leq g, \text { there exists a con- }\end{array}$ & $\begin{array}{l}\text { fying } f \leq g, \text { there exists a con- } \\
\text { tinuous real function } h \text { such that real function } h \text { such that }\end{array}$ \\
& $f \leq h \leq g$. & $f \leq h \leq g$. \\
\hline
\end{tabular}

Table 2: Characterizations of normal and extremally disconnected locales

It is our aim in this talk to discuss this parallel. We will present some results of our recent preprint [5] that provide positive answers to the following questions:

(1) There is a great variety of classical insertion type results (for several variants of normality). Can one unify them under a single general result?

(2) The proofs of the results in each pair are very different in nature. Can one unify them under the same result with a single proof?

We address these questions in the pointfree setting, that is, in the category of locales and localic maps (and its opposite category of frames and frame homomorphisms, objects of study of pointfree topology). For that we need to recall that sublocale lattices are more complicated than their topological counterparts (complete atomic Boolean algebras): they are precisely co-frames (i.e., the dual lattice of a frame).

Given a locale (frame) $L$, we denote by $\mathcal{S}(L)$ the dual of its sublocale lattice, which is a frame. In spite of being no longer a Boolean algebra, there are still plenty of complemented elements, among them the so-called closed sublocales $\mathfrak{c}(a)$ and the open sublocales $\mathfrak{o}(a)$ (for each $a \in L)$, complemented to each other. Furthermore, the map $a \mapsto \mathfrak{c}(a)$ is a frame embedding $L \hookrightarrow \mathcal{S}(L)$ providing an isomorphism $\mathfrak{c}: L \rightarrow \mathfrak{c}(L)$ between $L$ and the subframe $\mathfrak{c}(L)$ of $\mathcal{S}(L)$ of all closed sublocales. On the other hand, denoting by $\mathfrak{o}(L)$ the sublattice of $\mathcal{S}(L)$ formed by all $\mathfrak{o}(a)$, the correspondence $a \mapsto \mathfrak{o}(a)$ establishes a dual lattice embedding $L \rightarrow \mathfrak{o}(L)$.

Recall that a frame $L$ is normal if $a \vee b=1$ implies the existence of $u, v \in L$ such that $u \wedge v=0$ and $a \vee u=1=b \vee v$. This is a conservative extension of the classical notion: a space $X$ is normal iff the frame $\mathcal{O} X$ of open sets is normal. Using isomorphism $\mathfrak{c}$, this means that:

$$
\mathfrak{c}(a) \vee \mathfrak{c}(b)=1 \Rightarrow \exists u, v \in L: \mathfrak{c}(u) \wedge \mathfrak{c}(v)=0 \text { and } \mathfrak{c}(a) \vee \mathfrak{c}(u)=1=\mathfrak{c}(b) \vee \mathfrak{c}(v) .
$$

On the other hand, a frame $L$ is extremally disconnected (also De Morgan) if it is a Stone algebra, that is, $a^{*} \vee a^{* *}=1$ for every $a \in L$. (Again, we observe that this notion is in accordance with the corresponding classical one for a topological space $X$ : a space $X$ is extremally disconnected iff $\mathcal{O} X$ is extremally disconnected.) It can be easily shown that this is equivalent 
to

$$
\mathfrak{c}(a) \wedge \mathfrak{c}(b)=0 \Rightarrow \exists u, v \in L: \mathfrak{c}(u) \vee \mathfrak{c}(v)=1 \text { and } \mathfrak{c}(a) \wedge \mathfrak{c}(u)=0=\mathfrak{c}(b) \wedge \mathfrak{c}(v),
$$

which shows that normality and extremal disconnectedness notions are indeed dual to each other The following is also obvious: $L$ is normal (resp. extremally disconnected) iff $\mathfrak{c}(L)$ is normal (resp. extremally disconnected) iff $\mathfrak{o}(L)$ is extremally disconnected (resp. normal).

Now, let $B(\mathcal{S}(L))$ denote the Boolean part of $\mathcal{S}(L)$ (that is, the Boolean algebra of complemented elements of $\mathcal{S}(L))$. Fix an $\mathscr{A} \subseteq B(\mathcal{S}(L))$ and let $\mathscr{A}^{\mathrm{c}}$ denote the corresponding set of complements. Inspired by the definitions above, we introduce dual relative notions of normality and extremal disconnectedness, depending on the parameter $\mathscr{A}$ :

Definition. A frame $L$ is $\mathscr{A}$-normal if

for any $A, B \in \mathscr{A}$ such that $A \vee B=1$ there are $U, V \in \mathscr{A}$ such that $U \wedge V=0$ and $A \vee U=1=B \vee V$.

Dually, we say that $L$ is $\mathscr{A}$-disconnected if it is $\mathscr{A}^{\mathrm{c}}$-normal, that is, if

for any $A, B \in \mathscr{A}$ such that $A \wedge B=0$ there are $U, V \in \mathscr{A}$ such that $U \vee V=1$ and $A \wedge U=0=B \wedge V$.

By varying the choice of $\mathscr{A}$, we reach a wide array of examples. For example, classes $2^{2}$

$$
\begin{array}{ll}
\mathscr{A}_{1}=\mathfrak{c}(L), & \mathscr{A}_{2}=\left\{\mathfrak{c}\left(a^{*}\right) \mid a \in L\right\}, \\
\mathscr{A}_{4}=B(\mathcal{S}(L)), & \mathscr{A}_{5}=\{\mathfrak{c}(a) \mid a \text { is } \delta \text {-regular }\}
\end{array} \quad \mathscr{A}_{3}=\{\mathfrak{c}(a) \mid a \in \operatorname{Coz} L\},
$$

lead to the following notions 3 .

\begin{tabular}{lll}
\hline $\mathscr{A}$ & $\mathscr{A}$-normal frames & $\mathscr{A}$-disconnected frames \\
\hline $\mathscr{A}_{1}$ & normal frames & extremally disconnected frames \\
$\mathscr{A}_{2}$ & mildly normal frames & extremally disconnected frames \\
$\mathscr{A}_{3}$ & frames & $F$-frames \\
$\mathscr{A}_{4}$ & frames & frames \\
$\mathscr{A}_{5}$ & $\delta$-normal frames & extremally $\delta$-disconnected frames \\
\hline
\end{tabular}

Definition. We say that a real-valued function $f: \mathfrak{L}(\mathbb{R}) \rightarrow \mathcal{S}(L)$ on $L$ is lower $\mathscr{A}$-semicontinuous (resp. upper $\mathscr{A}$-semicontinuous) if for each $p<q$ in $\mathbb{Q}$ there exists $F_{p, q} \in \mathscr{A}$ such that

$$
f(q,-) \leq F_{p, q} \leq f(p,-) \quad\left(\text { resp. } f(-, p) \leq F_{p, q} \leq f(-, q)\right) .
$$

Then $f$ is $\mathscr{A}$-continuous if it is both lower and upper $\mathscr{A}$-semicontinuous. Again, these definitions bring to pointfree topology and unify several fundamental classical types of semicontinuities and continuities [Baire 1899, Dilworth 1950, Stone 1949, Lane 1983]:

\footnotetext{
${ }^{1}$ They are also formulable in any lattice and so one may speak more generally about normal and extremally disconnected lattices; evidently, a lattice is normal iff its dual lattice is extremally disconnected.

${ }^{2}$ Note that $\mathscr{A}_{1}, \mathscr{A}_{3}, \mathscr{A}_{4}$ and $\mathscr{A}_{5}$ are clearly sublattices of $B(\mathcal{S}(L))$ while $\mathscr{A}_{2}$ is only closed under finite meets.

${ }^{3}$ In case $L$ is $\mathcal{O} X$ for some space $X$, since every complemented sublocale of a space is a subspace, these notions are completely formulated in terms of the space $X$ (with no reference to sublocales) and provide a unification of several fundamental variants of normal and disconnected topological spaces in the literature [Blatter \& Seever 1972, Dilworth 1950, Katětov 1951, Lane 1975, 1979, 1983, Mack 1970, Seever 1968, Singal \& Singal 1973, Tong 1952 , etc.].
} 


\begin{tabular}{llll}
\hline $\mathscr{A}$ & lower $\mathscr{A}$-semicontinuous & upper $\mathscr{A}$-semicontinuous & $\mathscr{A}$-continuous \\
\hline $\mathscr{A}_{1}$ & lower semicontinuous & upper semicontinuous & continuous \\
$\mathscr{A}_{2}$ & normal lower semicontinuous & normal upper semicontinuous & normal continuous \\
$\mathscr{A}_{3}$ & zero lower semicontinuous & zero upper semicontinuous & zero continuous \\
$\mathscr{A}_{4}$ & $\mathrm{C}(B(\mathcal{S}(L)))$ & $\mathrm{C}(B(\mathcal{S}(L)))$ & $\mathrm{C}(B(\mathcal{S}(L)))$ \\
$\mathscr{A}_{5}$ & regular lower semicontinuous & regular upper semicontinuous & regular continuous \\
\hline
\end{tabular}

Further, we say that two sublocales $S$ and $T$ of $L$ are completely $\mathscr{A}$-separated if there is an $\mathscr{A}$-continuous function $f$ such that $f(0,-) \leq S$ and $f(-, 1) \leq T$.

A central tool for the proofs is a Katětov relation on $\mathcal{S}(L)$. Recall that a binary relation $\Subset$ on a lattice $M$ is a Katětov relation [1] if it satisfies the following conditions for all $a, b, a^{\prime}, b^{\prime} \in M$ :

(K1) $a \Subset b \Rightarrow a \leq b$.

(K2) $a^{\prime} \leq a \Subset b \leq b^{\prime} \Rightarrow a^{\prime} \Subset b^{\prime}$.

$(\mathrm{K} 3) a \Subset b$ and $a^{\prime} \Subset b \Rightarrow\left(a \vee a^{\prime}\right) \Subset b$.

(K4) $a \Subset b$ and $a \Subset b^{\prime} \Rightarrow a \Subset\left(b \wedge b^{\prime}\right)$.

(K5) $a \Subset b \Rightarrow \exists c \in M: a \Subset c \Subset b$.

Given a fixed $\mathscr{A} \subseteq B(\mathcal{S}(L))$, the relation $\Subset_{\mathscr{A}}$ on $\mathcal{S}(L)$ defined by

$$
S \Subset_{\mathscr{A}} T \equiv \exists U \in \mathscr{A}, \exists V \in \mathscr{A}^{\mathrm{c}}: S \leq V \leq U \leq T
$$

satisfies properties (K1) and (K2) and it is interpolative (i.e. satisfies (K5)) iff $L$ is $\mathscr{A}$-normal. We say that $\mathscr{A}$ is a Katětov class in $L$ whenever $\Subset_{\mathscr{A}}$ also satisfies conditions (K3) and (K4). Therefore, each Katětov class $\mathscr{A}$ in any $\mathscr{A}$-normal frame induces a Katětov relation $\Subset_{\mathscr{A}}$.

Examples of Katětov classes are:

- Any sublattice of $B(\mathcal{S}(L))$ (such as $\mathscr{A}_{1}, \mathscr{A}_{3}, \mathscr{A}_{4}$ and $\mathscr{A}_{5}$ ).

- Any $\mathscr{A} \subseteq B(\mathcal{S}(L))$, like $\mathscr{A}_{2}$, closed under binary joins and satisfying

$$
U_{1}, U_{2} \in \mathscr{A}, U_{1} \wedge U_{2} \geq V \in \mathscr{A}^{\mathrm{c}} \Rightarrow \exists U \in \mathscr{A}: U_{1} \wedge U_{2} \geq U \geq V .
$$

In this case, if $L$ is $\mathscr{A}^{\mathrm{c}}$-normal then $\mathscr{A}^{\mathrm{c}}$ is also a Katětov class.

Our main result characterizes $\mathscr{A}$-normal locales and generalizes the characterizations in Table 2 .

Theorem. [RELATIVE VERSION OF KATĚTOV-TONG INSERTION THEOREM] Let $\mathscr{A} \subseteq B(\mathcal{S}(L))$ be a Katětov class. The following are equivalent:

(i) $L$ is $\mathscr{A}$-normal.

(ii) If $f \leq g$ are real functions on $L$ such that $f$ is upper and $g$ is lower $\mathscr{A}$-semicontinuous, then there exists an $\mathscr{A}$-continuous real function $h$ on $L$ such that $f \leq h \leq g$.

(iii) Every $S, T \in \mathscr{A}$ satisfying $S \vee T=1$ are completely $\mathscr{A}$-separated. 
Then the dual result for $\mathscr{A}$-disconnectedness corresponds to the result for the class $\mathscr{A}^{\mathrm{c}}$ of complements of elements of $\mathscr{A}$ and is therefore obtained with no extra cost:

Corollary. [RELATIVE VERSION OF STONE INSERTION THEOREM]

Let $\mathscr{A} \subseteq B(\mathcal{S}(L))$ be such that $\mathscr{A}^{\mathrm{c}}$ is a Katětov class. The following are equivalent:

(i) $L$ is $\mathscr{A}$-disconnected.

(ii) If $g \leq f$ are real functions on $L$ such that $f$ is upper and $g$ is lower $\mathscr{A}$-semicontinuous, then there exists an $\mathscr{A}$-continuous real function $h$ on $L$ such that $g \leq h \leq f$.

(iii) Every $S, T \in \mathscr{A}$ satisfying $S \wedge T=0$ are completely $\mathscr{A}$-separated.

This approach extends and unifies the most relevant classical insertion results [Blatter \& Seever 1972, Katětov 1951, Tong 1952, Lane 1979, Stone 1949, etc.].

We will also discuss the conditions for $\mathscr{A}$ under which a relative version of Tietze's extension lemma holds (for that we need first to relativize the notion of an extension of a real function on a sublocale to the whole locale): we say that a Katětov class $\mathscr{A}$ of a locale $L$ is a Tietze class whenever

(T1) $\mathscr{A}$ is closed under finite meets, and (T2) $\mathscr{A}$ is closed under countable joins.

(Each of the guiding examples $\mathscr{A}_{1}, \mathscr{A}_{3}$ and $\mathscr{A}_{5}$ is a Tietze class.)

Theorem. [RELATIVE Version of Tietze's eXtension lemma] Let $\mathscr{A}$ be a Tietze class of $L$. Then $L$ is $\mathscr{A}$-normal iff every $S \in \mathscr{A}$ is $C_{\mathscr{A}}^{*}$-embedded in $L$.

Once again the dual result for extremal $\mathscr{A}$-disconnectedness follows immediately by complementation:

Corollary. [RELATive VERSion of Stone's EXTENSION LEMma] Let $\mathscr{A} \subseteq B(\mathcal{S}(L))$ be such that $\mathscr{A}^{\mathrm{c}}$ is a Tietze class. Then $L$ is $\mathscr{A}$-disconnected iff every $S \in \mathscr{A}^{\mathrm{c}}$ is $C_{\mathscr{A}}^{*}$-embedded in $L$.

If time permits, we will also present the conditions for $\mathscr{A}$ under which a relative version for the preservation of normality under localic maps holds. This extends the Hausdorff mapping invariance type theorems of Table 1 to the pointfree setting, thus completing Table 2

\section{References}

[1] J. Gutiérrez García and T. Kubiak, General insertion and extension theorems for localic real functions, J. Pure Appl. Algebra 215 (2011) 1198-1204.

[2] J. Gutiérrez García, T. Kubiak and J. Picado, Lower and upper regularizations of frame semicontinuous real functions, Algebra Universalis 60 (2009) 169-84.

[3] J. Gutiérrez García, T. Kubiak and J. Picado, Localic real-valued functions: a general setting, J. Pure Appl. Algebra 213 (2009) 1064-1074.

[4] J. Gutiérrez García and J. Picado, On the algebraic representation of semicontinuity, J. Pure Appl. Algebra 210 (2007) 299-306.

[5] J. Gutiérrez García and J. Picado, On the parallel between normality and extremal disconnectedness, preprint DMUC 13-04, March 2013, 37 pages (available at http://www. mat.uc.pt/ picado/preprint). 